\title{
A new approach for breast abnormality detection based on thermography
}

\author{
Type of article: Original article.
}

\author{
Nabil Karim Chebbah ${ }^{1}$, Mohamed Ouslim² ${ }^{2}$, Ryad Temmar ${ }^{1}$
}

1.PhD Student in Biomedical engineering and embedded system, LMSE laboratory, Department of Electronics,Faculty of Electrical Engineering, University of Science and Technology of Oran, Oran, Algeria

2. Professor, LMSE laboratory, Department of Electronics, Faculty of Electrical Engineering, University of Science and Technology of Oran (USTO), Oran, Algeria chebbah.nabilkarim@gmail.com

\begin{abstract}
Background: Breast cancer is one of the most common women cancers in the world. In this paper, a new method based on thermography for the early detection of breast abnormality is proposed.

Methods: The study involved 80 breast thermograms collected from the PROENG public database which consist of 50 healthy breasts and 30 with some findings. Image processing techniques such as segmentation, texture analysis and mathematical morphology were used to train a support vector machine (SVM) classifier for automatic detection of breast abnormality.

Results: After conducting several tests, we obtained very interesting and motivating results. Indeed, our classifier showed high performance results giving an accuracy of $91.25 \%$, a sensitivity and a specificity of $93.3 \%$ and $90 \%$, respectively.

Conclusion: The final results let us conclude that infrared thermography with the help of an adequate automatic classification algorithm can be a valuable and reliable complementary tool for radiologists in detecting breast cancer and thereby helping to reduce mortality rates.
\end{abstract}

Keywords: Breast cancer, Thermography, Image processing, Computer-Assisted Diagnosis

Corresponding author: Nabil Karim Chebbah, Biomedical engineering and embedded system, LMSE laboratory, Department of Electronics,Faculty of Electrical Engineering, University of Science and Technology of Oran, Oran, Algeria. Email: chebbah.nabilkarim@gmail.com

Received: June 30, 2018, Accepted: September 2, 2018, English editing: September 28, 2018, Published: September 30, 2018.

Screened by iThenticate.@2017-2018 KNOWLEDGE KINGDOM PUBLISHING.

\section{Introduction}

Nowadays, breast cancer is the most common invasive cancer and the second leading cause of cancer death for women worldwide. The WHO (World Health Organization) assess that more than one million new cases are diagnosed each year and over 600000 deaths are related to breast cancer across the world [1]. 
In Algeria, The Cancer Registry of Set if in collaboration with the IARC (International Agency for Research on Cancer) list breast cancer as the highest identified cancer cases in Algeria, with more than 11000 new cases and 3000 deaths each year and its incidence continues to increase by $7 \%$ a year, making it a major public health problem [2].

The improvement of advanced screening tools for the early breast cancer detection becomes essential to reduce the mortality rate. Previous researchers showed that there is an $85 \%$ chance of cure if the tumor is detected in a precocious stage and only a $10 \%$ chance if detected later [3].Hence, it is a very useful task to provide diagnostic tools with high accuracy to help radiologists in reducing false positive predictions.

Currently, there are several techniques to detect and provide a diagnosis of breast abnormalities, such as echography, tomosynthesis, Magnetic Resonance Imaging (MRI), mammography, Positron Emission Tomography (PET) and breast thermography. Each of them has well known advantages and disadvantages. Mammography is the first line imaging technique for breast cancer detection. It is a radiological screening modality that uses an X-ray beam to detect anatomical changes in breast tissues. However, recent research has suggested that mammography cannot be considered an ideal tool for early diagnosis [3]. For example, the tumor must at least exceed a certain thickness to be identified. Indeed, in [4], the authors reported that the size of the tumor less than $1.66 \mathrm{~cm}$ was very difficult to detect by mammography. On the other hand, this technique also exposes patients to X-rays that can damage tissue and cause mutations [3].

Due to the limitations of the current imaging techniques, researchers have given special attention to thermography as a screening tool for breast cancer over the last two decades due to the fact that this procedure is non-ionizing, non-invasive and can detect breast abnormalities at an early stage. This technique has been widely accepted as an auxiliary screening tool for breast cancer detection to quantify risk and identify the population who will benefit from more detailed oncological tests [5].

\section{Previous related work}

More than 2000 years ago, Hippocrates used the first thermo-biological application for a pathological diagnosis. He applied mud all over a patient's body and then he noticed that the mud dried quicker in some places, which helped him identify the unhealthy areas [6].Since then, many studies have focused onthermobiology realizing that cancerous area in the mammary organ produces a significantly higher temperature than healthy breasts. This is because these lesions (or tumors) contain more veins and have a higher metabolic rate than the surrounding tissues [7]. In the research conducted by Professor Gautherie, at the laboratory of biomedical thermology at Louis Pasteur University [8], 784 subjects with initially normal breasts (with no mammographics or echographics findings) and anomalous thermograms were carefully attended during a period of twelveyear. Subsequently, 238 patients developed cancer along the 4 years that followed, which represents $38 \%$ of the patients who were initially diagnosed as abnormal by 
thermography and normal by mammography. Therefore, researchers stated that IR thermography could predict the development of breast cancer quite effectively.
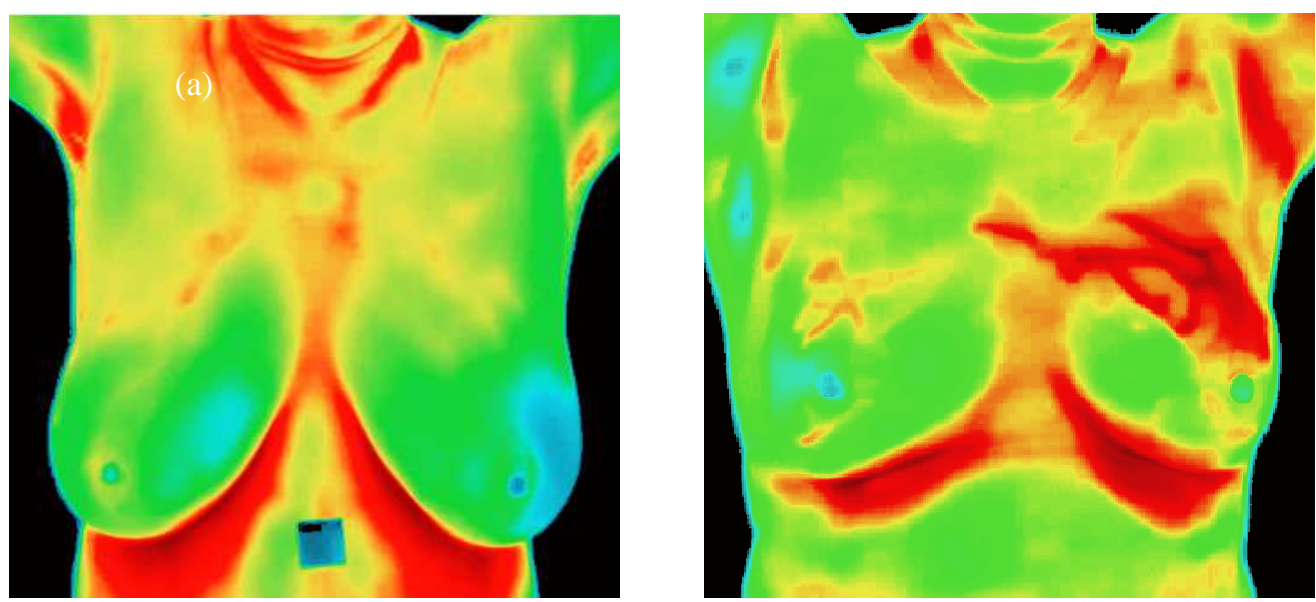

Figure 1. Breast thermogram: (a) Shows healthy breasts (b) Shows advancedcancer in the left breast

More details on the thermography mechanism, the protocol acquisition, and patient characteristic are found in [6][9][10].

Although thermography is a promising screening tool for breast cancer, a robust and efficient computer-aided diagnosis (CAD) system must be developed to overcome factors such as scarcity of qualified personnel and limitation of the human visual system to differentiate the minimal temperature differences produced by thermographic imaging. Several researchers have worked on developing CAD systems for breast cancer detection based on advances in digital image processing and data mining. Sheeja et al. suggest the use of curvelet based feature extraction in order to classify breast thermograms as normal or abnormal; the proposed approach showed $90.91 \%$ accuracy, $81.82 \%$ and $100 \%$ of sensitivity and specificity, respectively [4]. Rajendra et al. extracted texture features using structural and statistical approach with a support vector machine (SVM) classifier to automatically detect signs of breast cancer; 50 thermographic images were used (25 normal and 25 cancerous);88.10\% of sensitivity, $85.71 \%$ of specificity and $90.48 \%$ of accuracy were obtained [11].Schaefer et al. performed a fuzzy-logic based classifier coupled with a variety of statistical features based on the asymmetry analysis of the breast and providing a classification accuracy of 80\%[12]. Pramanik et al. presented an automatic diagnosis system based on discrete wavelet transform to detect early breast cancer in a thermogram; the Otsu threshold and morphological operation-based segmentation were applied to define the ROI; then, statistical and texture features were extracted and used to train an artificial neural network classifier providing an accuracy of 90.48\%[13].Acharya et al. investigated the conversion of the $2 \mathrm{D}$ thermograms into a one-dimensional data using a radon transform; high order spectral features were extracted from the transformed data in order to analyze the asymmetry between the two breasts; the methodology obtained $80 \%$ accuracy, $76 \%$ and $84 \%$ sensitivity and specificity, 
respectively using the SVM classifier. However, in [14] artificial neural network classifier gave better performance values with $92 \%$ of sensitivity, $88 \%$ of specificity, and $90 \%$ of accuracy. Ming et al. [15]developed a CAD system based on feature extraction and decision tree classifier for the automatic detection of breast cancer. Tuan et al. investigated the usage of complementary learning fuzzy neural networks for breast cancer classification; the authors achieved an accuracy of $86.6 \%$ [16]. Gerald. S presented an approach of analyzing breast thermograms that used features extraction and ant colony optimization (ACO) based pattern recognition for the classification of normal and abnormal breast thermogram; experimental results gave an accuracy of $79.52 \%$ [17]. Kandlikar et al. reviewed the advances over the last three decades in the use of thermal imaging to detect breast cancer [7].

\section{The proposed work}

In this study, we propose a new approach for a computer aided diagnosis system based on breast thermography to decrease the operator dependence and to assist radiologists in the precocious breast cancer detection. Figure 2 illustrates the process plan of the main operations involved in the proposed technique.

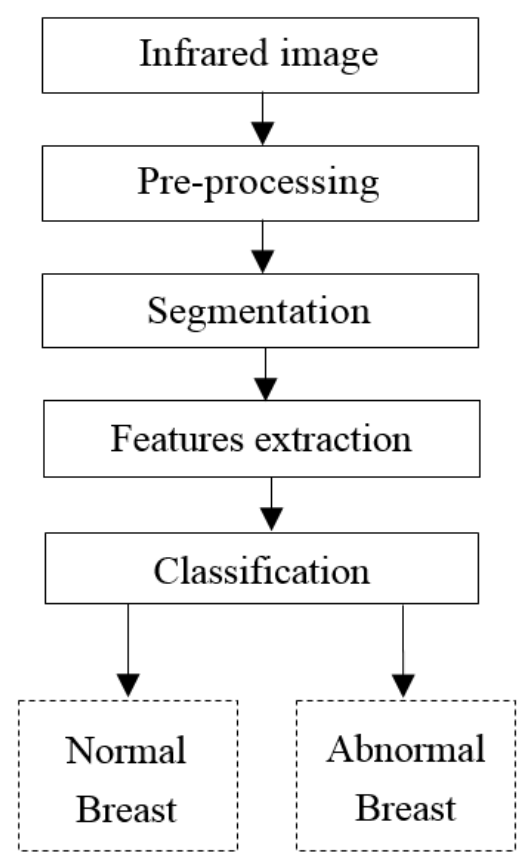

Figure 2. Proposed block diagram

After the image acquisition, we converted the breast thermograms from color to gray scale image, then the regions of interest (ROIs) were segmented, which involve the separation areas of the image that represent the breast from the background and isolate the left and right breasts; then, a GLCM (Gray level cooccurrence matrix) and morphological operations were used on the segmented image to extract statistical and textural features. Finally, a supervised learning technique, namely SVM (Support Vector Machine) was performed to classify 
breasts as normal or abnormal. The remainder part of this paper explains in more details all the steps involved within the proposed technique.

\subsection{Data acquisition}

In this study, a total of 80 frontal breast thermograms 50 normal and 30 abnormal were collected from an open source online database [18]. Infrared thermograms were acquired at the Fluminense Federal University (UFF) using a FLIR Therma Cam SC620 infrared camera with a spectral bandwidth of $7.5-13 \mu \mathrm{m}$ (30 frames/s), a thermal resolution of $40 \mathrm{mK}$ at $30^{\circ} \mathrm{C}$ and an image resolution of $640 \times 480$ pixels. All the patients with abnormal breast were previously diagnosed through biopsies and their thermography report was confirmed by a skilled specialist [18].

\subsection{Preprocessing and segmentation}

The first stage of the CAD system is preprocessing. This includes image resizing and converting the RGB color input image into intensity gray scale in which white pixels will represent the highest temperatures and black, the lowest ones. This step of image processing is used to enhance the quality of the thermographic images and make the classification and features extraction phases more efficient.

In the segmentation stage, unnecessary details that may lead to wrong diagnosis such as neck, inframammary folds and background have been removed and the region of interest has been separated into right and left breast. The classification performance results depend greatly on the proper delineating of the two breasts. For this purpose, segmentation was carried out manually in this work with medical expert's assistance to obtain the most exact delineating of the right and left breast due to absence of definite shape and clear edges of the breast in thermal images [19]. Various segmentation algorithms have been proposed to delineate the breast automatically or semi-automatically, but with moderate success rates [20][21][22][23].Figure 3 shows the resulting right and left breast thermogram after preprocessing and segmentation phases.

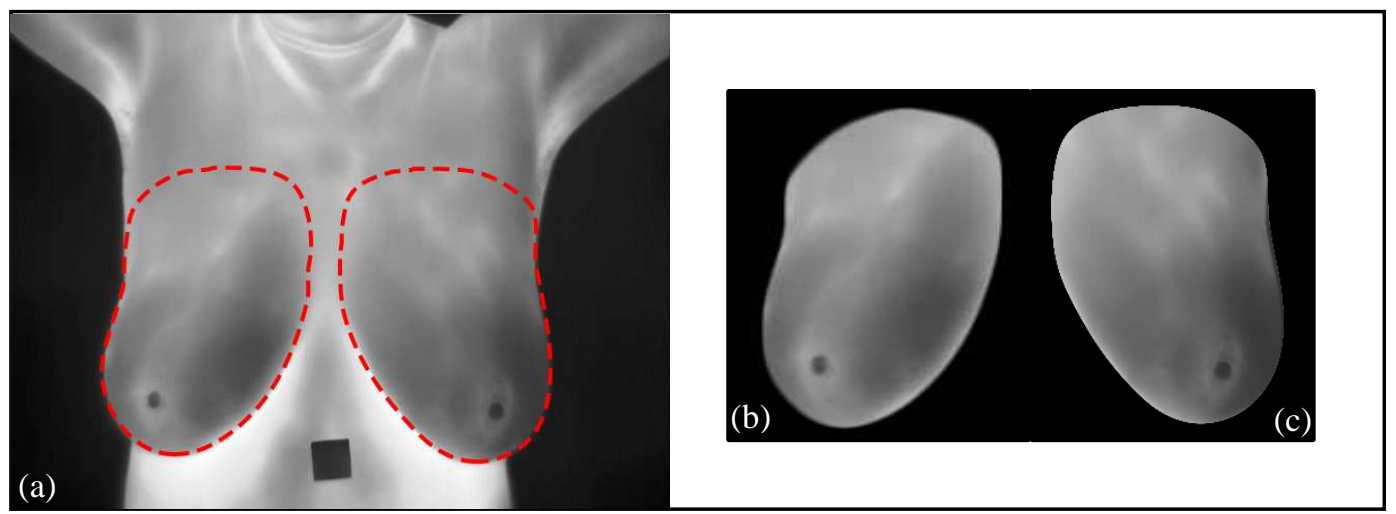

Figure 3.(a)Resulting grayscalethermogram (b) cropped right breast (c) cropped left breast 


\subsection{Features extraction}

This step of CAD system represents an important image processing task by which certain characteristics of interest in an image are converted from a graphical representation (data in pixels) to a quantitative representation (data in vector).

In this third phase of CAD system, features are extracted using texture analysis, which represent the thermal variations of the breastquite effectively. Several methods have been proposed to measure texture properties of an image [24], one of these methods is the GLCM (Gray Level Co-occurrence Matrix)which describes the texture of an image by calculating how frequently combinations of pixels with a definite value of gray intensity and in a specific dimension occur in an image[24].In this work we computed and normalized GLCM of the ROI, a series of second order statistical texture parameters proposed by Haralick [25] are then extracted from this matrix. Besides, some first and high order statistical measures calculated from the original image values were added with the previous features in order to improve the classification accuracy.

\subsection{Classification}

Feature vectors extracted from segmented breast thermograms are used to form an SVM classifier that proved good performance for the automatic detection of cancerous breast. Several supervised learning methods have been reported in the literature for breast cancer detection based on thermography such as: Artificial Neural Network [13], K-nearest neighbors [26], fuzzy logic-based classifier [12] and Support Vector Machine (SVM) [11].The latter is the most powerful and it is widely used in the classification of health diseases, especially in oncology, due to its high accuracy and its ability to deal with high-dimensional data.

\section{Results and comments}

We conducted several tests by applying the proposed method on the large dataset of 80 breast thermograms. In order to measure the method's performance, we selected three metrics: the sensitivity, the specificity and the accuracy.

Sensitivity is the possibility that a test used in a sick patient will produce a positive result.It is given by the equation (1).

$$
\text { Sensitivity }=\frac{\text { true positive }}{\text { true positive }+ \text { false negative }}
$$

Specificity is the possibility that a test used in a healthy patient will produce a negative result. The formula for finding specificity is given in the equation (2).

$$
\text { Specificity }=\frac{\text { true negative }}{\text { true negative }+ \text { false positive }}
$$


Accuracy is defined as the number of correct predictions from all predictions made. It is given by the equation (3).

Accuracy $=\frac{\text { true positive }+ \text { true negative }}{\text { true positive }+ \text { false positive }+ \text { true negative }+ \text { true negative }}$

The obtained experimental results are summarized in Table 1 where we can clearly see the excellent classification performance of the proposed method.

Table 1 Classification performance

\begin{tabular}{cc}
\hline $\begin{array}{c}\text { Performance } \\
\text { measures }\end{array}$ & $\begin{array}{c}\text { SVM } \\
\text { Performance }\end{array}$ \\
\hline Sensitivity & $\mathbf{9 3 . 3 \%}$ \\
\hline Specificity & $\mathbf{9 0} \%$ \\
\hline Accuracy & $\mathbf{9 1 . 2 5 \%}$ \\
\hline
\end{tabular}

We also present in Table 2 the confusion matrix, for further inspection of the testing approach.

\begin{tabular}{|c|c|c|c|}
\hline \multirow{2}{*}{$\begin{array}{c}\text { Table 2Confusion matrix of } \\
\text { the classification results }\end{array}$} & \multicolumn{2}{|c|}{ Actual class } \\
\cline { 2 - 4 } \\
$\begin{array}{c}\text { Predicted } \\
\text { class }\end{array}$ & Abnormal & 28 True positive & 5 False positive \\
\cline { 2 - 4 } & Normal & 2False negative & 45True negative \\
\hline
\end{tabular}

All the tests were carried out on Intel core i7-5500U 2.4GHz processor with 8GB RAM, running under Windows 7 64-bits operating system. The averaged computation time for a 200x200 size image was around 0.14 seconds. The algorithm and a graphical user interface shown in Figure 4 were developed via Matlab 2016.

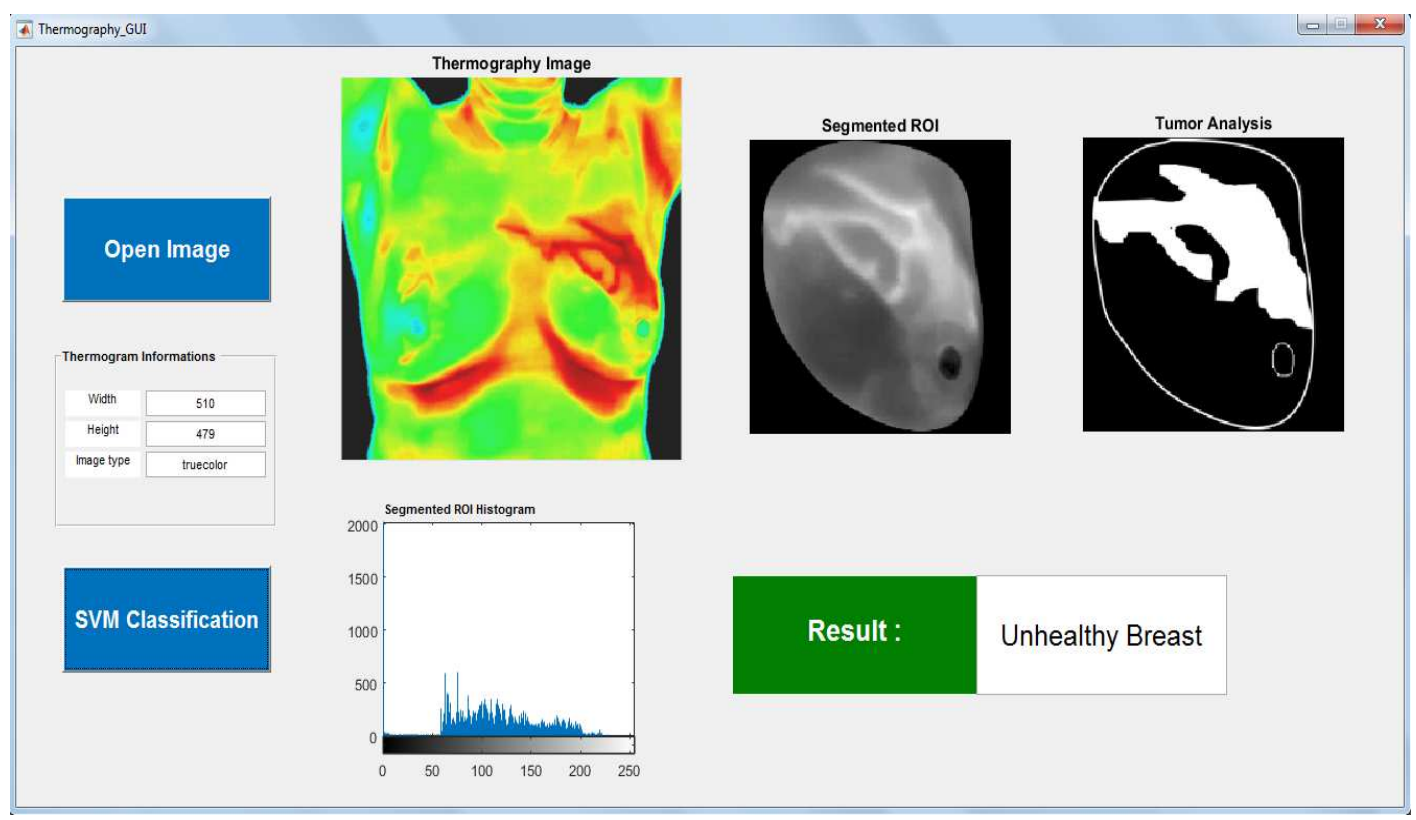


Figure 4.Menu driven interface of the presented CAD system

Different functionalities have been introduced to facilitate the use of this graphical interface. The Open Image button is used to load the breast thermography as well as the corresponding segmented region of interest. The Image information panel shows details of the input thermogram, such as width, height and image type. The software also displays the histogram of the segmented ROI. In the other hand, by clicking on the SVM classification button, the feature extraction operation is automatically done followed by their feeding in the support vector machine classifier. Finally, the system returns the result of the classification that is displayed in the results section. In the figure above, the result shows that the input segmented thermogram corresponds to an unhealthy breast class.

\section{Conclusion and Further development}

The present paper suggests an automatic computer assisted diagnostic approach for the precocious breast cancer detection based on medical thermal imaging. The region of interest has been converted to grayscale and separated from the background and extra region. Subsequently, both textural and statistical features were extracted to feed a supervised learning machine for the classification of unhealthy and normal breast thermograms. Experimental results affirm the effectiveness of the method, giving a $93.3 \%$ of sensitivity, $90 \%$ of specificity and $91.25 \%$ of accuracy. We conclude that breast thermography is a good screening modality for the early detection of breast cancer and should be implemented as a complementary test in our country. As a continuation to the work, we propose the implementation of this method on an embedded system to take advantage of the parallelism available in some architectures in order to improve the system performances and reduce the computation time.

\section{Acknowledgement}

Authors thank Dr F Senouci from Oran Healthcare Center, Algeria, for the assistance in the segmentation of the regions of interest.

\section{Conflict of intereststatement}

We certify that there is no conflict of interest with any financial organization in the subject matter or materials discussed in this manuscript.

\section{Authors' biography}

No Biography

\section{Reference}

[1] Ibrahim, Abdelhameed, Shaimaa Mohammed, and Hesham Arafat Ali. "Breast Cancer Detection and Classification Using Thermography: A Review." International Conference on Advanced Machine 
Learning Technologies and Applications. Springer, Cham, (2018). https://doi.org/10.1007/978-3-319-74690-6 49

[2] Hamdi-Cherif, M., et al. "Cancer estimation of incidence and survival in Algeria 2014." J Cancer $\begin{array}{llll}\text { Res } & \text { Ther } & 3.9 & \text { (2015): }\end{array}$ https://doi.org/10.14312/2052-4994.2015-14

[3] Lanisa, Norlailah, Ng Siew Cheok, and Lai Khin Wee. "Color morphology and segmentation of the breast thermography image." Biomedical Engineering and Sciences (IECBES), 2014 IEEE Conference on. IEEE, (2014).

[4] Francis, Sheeja V., M. Sasikala, and S. Saranya. "Detection of breast abnormality from thermograms using curvelet transform based feature extraction." Journal of medical systems 38.4 (2014): 23 .

[5] Lahiri, B. B., et al. "Medical applications of infrared thermography: a review." Infrared Physics \& $\begin{array}{lll}\text { Technology } & \text { (2012): } & \\ & & \end{array}$ https://doi.org/10.1016/.i.infrared.2012.03.007

[6] Etehadtavakol, Mahnaz, and Eddie YK Ng. "An Overview of Medical Infrared Imaging in Breast Abnormalities Detection." Application of Infrared to Biomedical Sciences. Springer, Singapore, (2017). 45-57.

[7] Yaneli, Ameca-Alducin Maria, et al. "Assessment of bayesian network classifiers as tools for discriminating breast cancer pre-diagnosis based on three diagnostic methods." Mexican International Conference on Artificial Intelligence. Springer, Berlin, Heidelberg, (2012).

[8] Gautherie, Michel, and Charles M. Gros. "Breast thermography and cancer risk prediction." $\begin{array}{lll}\text { Cancer } & 45.1 & \text { (1980): }\end{array}$ https://doi.org/10.1002/1097-0142(19800101)45:1<51::AID-CNCR2820450110>3.0.CO;2-L https://doi.org/10.1002/cncr.2820450110

PMid:7351006

[9] Kandlikar, Satish G., et al. "Infrared imaging technology for breast cancer detection-Current status, protocols and new directions." International Journal of Heat and Mass Transfer 108. USA (2017): 2303-2320.

[10] Vardasca, Ricardo, Lucia Vaz, and Joaquim Mendes. "Classification and Decision Making of Medical Infrared Thermal Images." Classification in BioApps. Springer, Cham, (2018). 79-104.

[11] Acharya, U. Rajendra, et al. "Thermography based breast cancer detection using texture features and support vector machine." Journal of medical systems 36.3 (2012): 1503-1510. https://doi.org/10.1007/s10916-010-9611-z

PMid:20957511

[12] Schaefer, Gerald, Michal Závišek, and Tomoharu Nakashima. "Thermography based breast cancer analysis using statistical features and fuzzy classification." Pattern Recognition 42.6 (2009): 1133-1137.

https://doi.org/10.1016/j.patcog.2008.08.007

[13] Pramanik, Sourav, Debotosh Bhattacharjee, and MitaNasipuri. "Wavelet based thermogram analysis for breast cancer detection." Advanced Computing and Communication (ISACC), 2015 International Symposium on. IEEE, (2015).

[14] Acharya, U. Rajendra, et al. "Higher order spectra analysis of breast thermograms for the automated identification of breast cancer." Expert Systems 31.1 (2014): 37-47. https://doi.org/10.1111/i.1468-0394.2012.00654.x

[15] Lee, Ming-Yih, and Chi-Shih Yang. "Entropy-based feature extraction and decision tree induction for breast cancer diagnosis with standardized thermograph images." Computer methods

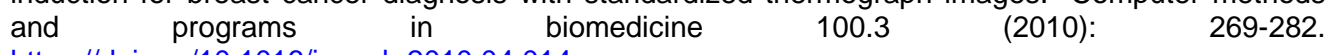
https://doi.org/10.1016/i.cmpb.2010.04.014

PMid:20537756

[16] Tan, Tuan Zea, et al. "A novel cognitive interpretation of breast cancer thermography with complementary learning fuzzy neural memory structure." Expert Systems with Applications 33.3 (2007):

https://doi.org/10.1016/i.eswa.2006.06.012

652-666.

[17] Gerald, S. "ACO classification of thermogram symmetry features for breast cancer diagnosis".

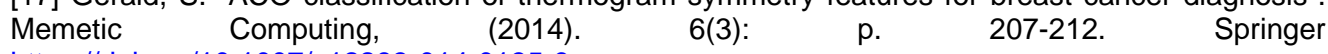
https://doi.org/10.1007/s12293-014-0135-9

[18] Silva, L. F., et al. "A new database for breast research with infrared image." Journal of Medical $\begin{array}{llll}\text { Imaging } \quad \text { Health Informatics } & 4.1 & \text { (2014): } & \text { 92-100. }\end{array}$ https://doi.org/10.1166/jmihi.2014.1226

[19] Borchartt, Tiago B., et al. "Breast thermography from an image processing viewpoint: A survey." $\begin{array}{llll}\text { Signal } & \text { Processing } & 93.10 & \text { (2013): }\end{array}$ https://doi.org/10.1016/.jsigpro.2012.08.012

[20] Suganthi, S. S., and S. Ramakrishnan. "Semi automatic segmentation of breast thermograms using variational level set method." The 15th International Conference on Biomedical Engineering. Springer, Cham, (2014).

[21] Golestani, N., M. EtehadTavakol, and E. Y. K. Ng. "Level set method for segmentation of infrared breast thermograms." EXCLI journal $13 \quad$ (2014): 241. PMid:26417258 PMCid:PMC4464455 
[22] Srinivasan, Suganthi Salem, and Ramakrishnan Swaminathan. "Segmentation of breast tissues in infrared images using modified phase based level sets." Biomedical Informatics and Technology. Springer, Berlin, Heidelberg, (2014). 161-174.

[23] De Oliveira, J. P. S., et al. "Segmentation of infrared images: A new technology for early detection of breast diseases." Industrial Technology (ICIT), 2015 IEEE International Conference on. IEEE, (2015).

[24] Shang, Zhigang, and Mengmeng Li. "Combined feature extraction and selection in texture analysis." Computational Intelligence and Design (ISCID), 2016 9th International Symposium on. Vol. 1. IEEE, (2016).

[25] Haralick, Robert M., and Karthikeyan Shanmugam. "Textural features for image classification." IEEE Transactions on systems, man, and cybernetics 6 (1973): 610-621. https://doi.org/10.1109/TSMC.1973.4309314

[26] Milosevic, Marina, Dragan Jankovic, and Aleksandar Peulic. "Thermography based breast cancer detection using texture features and minimum variance quantization." EXCLI journal 13 (2014):

1204.

PMid:26417334 PMCid:PMC4464488

[27] Rassiwala, Muffazzal, et al. "Evaluation of digital infra-red thermal imaging as an adjunctive screening method for breast carcinoma: A pilot study." International Journal of Surgery 12.12 (2014): 1439-1443.

https://doi.org/10.1016/.i.jsu.2014.10.010

PMid:25448668 